H. Tamura

Nagoya Math. J.

Vol. 105 (1987), 49-69

\title{
ASYMPTOTIC DISTRIBUTION OF EIGENVALUES FOR SCHRÖDINGER OPERATORS WITH MAGNETIC FIELDS
}

\author{
HIDEO TAMURA
}

\section{§. Introduction}

The asymptotic distribution of eigenvalues has been studied by many authors for the Schrödinger operators $-\Delta+V$ with scalar potential growing unboundedly at infinity. Let $N(\lambda)$ be the number of eigenvalues less than $\lambda$ of $-\Delta+V$ on $L^{2}\left(R_{x}^{n}\right)$. Under suitable assumptions on $V(x)$, $N(\lambda)$ obeys the following asymptotic formula:

$$
N(\lambda)=(2 \pi)^{-n} \operatorname{vol}\left[\left\{(x, \xi):|\xi|^{2}+V(x)<\lambda\right\}\right](1+o(1)), \quad \lambda \rightarrow \infty .
$$

This formula has been obtained by [10] for a wide class of potentials. See the survey [2] for comprehensive references. In the present work, we shall study the same problem for the Schrödinger operator

$$
H=(i \nabla+A(x) / 2)^{2}=\sum_{j=1}^{3}\left(i \partial_{j}+a_{j}(x) / 2\right)^{2}, \quad \partial_{j}=\partial / \partial x_{\jmath},
$$

with the magnetic vector potential $A(x)=\left(a_{1}(x), a_{2}(x), a_{3}(x)\right)$.

The leading term of the asymptotic formula for the case of scalar potentials can be determined by the phase space volume of the region $\left\{(x, \xi):|\xi|^{2}+V(x)<\lambda\right\}$. However, this is not the case for vector potentials, because the corresponding quantity

$$
\left.\operatorname{vol}\left\{(x, \xi): \sum_{j=1}^{3}\left(\xi_{j}+a_{j}(x) / 2\right)^{2}<\lambda\right\}\right]=\infty
$$

for any magnetic vector potential $A(x)$. Nevertheless, we know that the operator $H$ has only discrete spectrum under suitable assumptions on $A(x)$ ([4], [6]). In [13], Simon has called such problems non-classical eigenvalue asymptotics. Thus the asymptotic distribution of eigenvalues for the case of vector potentials is one of typical problems of non-classical eigenvalue asymptotics.

Received July 25, 1985. 
The Schrödinger operators with magnetic vector potentials which we here discuss are analogous to the hypoelliptic operators $\sum_{k=1}^{p} X_{k}^{*} X_{k}$ introduced by Hörmander [5], $X_{k}$ being real vector fields. The problem of spectral asymptotics has been studied by [8] for such operators defined on bounded regions.

\section{$\S 1$. Main theorems}

We work exclusively in the 3 -dimensional space $R_{x}^{3}$ and consider the operator

$$
H=(i \nabla+A(x) / 2)^{2}=\sum_{j=1}^{3}\left(i \partial_{j}+a_{j}(x) / 2\right)^{2}, \quad \partial_{j}=\partial / \partial x_{j} .
$$

We begin by making several assumptions on the magnetic vector potential $A(x)=\left(a_{1}(x), a_{2}(x), a_{3}(x)\right)$. First we assume that:

$$
a_{j}(x), 1 \leqq j \leqq 3 \text {, are real } C^{2} \text {-smooth functions } .
$$

By assumption (A.1), $H$ is essentially self-adjoint in $C_{0}^{\infty}\left(R_{x}^{3}\right)$ (Schechter [11]). We denote by the same notion $H$ the unique self-adjoint realization in $L^{2}\left(R_{x}^{3}\right)$. The magnetic field $B(x)$ is given by

$$
B(x)=\left(b_{1}(x), b_{2}(x), b_{3}(x)\right)=\nabla \times A(x) .
$$

Set

$$
b(x)=|B(x)|=\left(\sum_{j=1}^{3} b_{j}(x)^{2}\right)^{1 / 2} .
$$

The following the assumptions imply the compactness property of $(H+i)^{-1}$, so that $H$ has only discrete spectrum:

$$
\begin{gathered}
b(x) \rightarrow \infty \quad \text { as }|x| \rightarrow \infty ; \\
\left|\partial_{x}^{\alpha} a_{\jmath}(x)\right|=o\left(b(x)^{3 / 2}\right), \quad|\alpha|=2, \quad \text { as }|x| \rightarrow \infty .
\end{gathered}
$$

Here we should refer to the results of [4] and [6] on the compactness property of $(H+i)^{-1}$. Consider the condition

$$
\left|\nabla b_{j}(x)\right|=o\left(b(x)^{\rho}\right), \quad 1 \leqq j \leqq 3, \quad \rho>0, \quad \text { as }|x| \rightarrow \infty .
$$

The compactness of $(H+i)^{-1}$ has been proved by [4] under (A.2) and (B) with $\rho=3 / 2$ and by [6] under (A.2) and (B) with $\rho=2$. Furthermore, it has been shown that the compactness property does not follow from (A.2) only. In particular, Iwatsuka [6] has obtained that $\rho=2$ is the border line for the compactness, by constructing an example in which $b(x)$ satisfies $\left|\nabla b_{\jmath}(x)\right|=O\left(b(x)^{2}\right)$ but $(H+i)^{-1}$ is not compact. 
Let

$$
m(\lambda)=\operatorname{meas}[\{x: b(x)<\lambda\}] .
$$

Then the final assumption we make is that:

$$
m(2 \lambda) \leqq C m(\lambda), \quad C>1, \quad \text { for } \lambda>\Lambda_{0} \gg 1 .
$$

Theorem 1. Let $H$ be defined by (1.1). Assume (A.1)-(A.4) and denote by $N(\lambda)$ the number of eigenvalues less than $\lambda$ of $H$. Then

$$
N(\lambda)=2(2 \pi)^{-2} \theta(\lambda ; b)(1+o(1)), \quad \lambda \rightarrow \infty,
$$

where

$$
\theta(\lambda ; b)=\sum_{j=0}^{\infty} \int_{(2 j+1) b(x)<2} b(x)(\lambda-(2 j+1) b(x))^{1 / 2} d x .
$$

Next we shall discuss the problem on the semi-classical asymptotic distribution of eigenvalues. Consider the operator

$$
H_{h}=(i h \nabla+A(x) / 2)^{2}, \quad 0<h \leqq 1 .
$$

The semi-classical asymptotic formula as $h \rightarrow 0$ can be obtained under a little milder assumptions than the high energy asymptotic formula as $\lambda \rightarrow \infty$ (Theorem 1). We assume (A.1), (A.2), (A.4) and

$$
\left|\partial_{x}^{\alpha} a_{j}(x)\right|=o\left(b(x)^{2}\right), \quad|\alpha|=2, \quad \text { as }|x| \rightarrow \infty,
$$

in place of (A.3). Under (A.2) and (A.3'), $H_{h}$ has only discrete spectrum by the above result due to Iwatsuka [6].

Theorem 2. Let $H_{h}$ be defined by (1.5). Assume (A.1), (A.2), (A.3') and (A.4), and denote by $N_{E}(h), E>0$, the number of eigenvalues less than $E$ of $H_{h}$. Then

$$
N_{E}(h)=2(2 \pi)^{-2} \theta\left(E h^{-2} ; h^{-1} b\right)(1+o(1)), \quad h \rightarrow 0,
$$

where $\theta(\lambda ; b)$ is defined by (1.4).

We shall explain briefly why the asymptotic formula for $N_{E}(h)$ can be obtained under a little milder assumptions. As is easily seen, $N_{E}(h)$ coincides with the number of eigenvalues less than $E h^{-2}$ of the operator

$$
H(h)=\left(i \nabla+h^{-1} A(x) / 2\right)^{2} .
$$

From Theorem 1 we can guess that eigenfunctions associated with eigen- 
values less than $E h^{-2}$ of $H(h)$ are almost supported in the region $\{x: b(x)$ $\left.<E h^{-1}\right\}$. In this region, we have

$$
\left|\partial_{x}^{\alpha}\left(h^{-1} a_{j}(x)\right)\right| \leqq \varepsilon(x)\left(h^{-1} b(x)\right)^{3 / 2}, \quad|\alpha|=2,
$$

by assumption (A.3'), where $\varepsilon(x) \rightarrow 0$ as $|x| \rightarrow \infty$. This corresponds just to assumption (A.3) in Theorem 1. Thus (A.3) can be weakened slightly in the case of semi-classical eigenvalue asymptotics.

In [3], the asymptotic formula for $N_{E}(h)$ has been derived for operators of the form $(i \nabla+A(x))^{2}+V(x)$ with scalar potential $V(x)$ growing unboundedly at infinity. By making use of the min-max principle combined with the Dirichlet-Neumann bracketing and by assuming only the continuity of $A(x)$ and $V(x), N_{E}(h)$ has been proved to obey the following asymptotic formula:

$$
N_{E}(h)=(2 \pi h)^{-3} \operatorname{vol}\left[\left\{(x, \xi): \sum_{j=1}^{3}\left(\xi_{j}+a_{j}(x)\right)^{2}+V(x)<E\right\}\right](1+o(1)) .
$$

Thus the leading term is determined by the classical quantity. Roughly speaking, in the presence of growing potential $V(x)$, the eigenstates with eigenvalues less than $E$ are confined in the bounded region $\{x: V(x)<E\}$ uniformly in $h$. This makes the situation slightly easy to deal with. The min-max principle as in [3] does not seem to apply directly to the present case without growing potentials.

The proof of both Theorems 1 and 2 is based on the same method. We study the asymptotic behavior as $t \rightarrow 0$ of the trace $\operatorname{Tr}[\exp (-t H)]$ or $\operatorname{Tr}[\exp (-t H(h))]$ by use of the Feynman-Kac-Ito formula and apply the tauberian arguments due to Karamata [7] to the asymptotic formula obtained for the above trace. We give the detailed proof to Theorem 2 and only a sketch to Theorem 1 .

\section{§2. Reduction to main lemmas}

Throughout the entire discussion, the assumptions of Theorem 2 are assumed to be satisfied and, for brevity, we fix $E=1$ in Theorem 2 . Let $\Lambda_{0}$ be as in (A.4). Without loss of generality, we further assume that $0<h<\Lambda_{0}^{-1}$.

Let $H(h)$ be defined by (1.6). We denote by $\left\{\lambda_{j}(h)\right\}_{j=1}^{\infty}, 0<\lambda_{1} \leqq \lambda_{2} \leqq \cdots$, a sequence of eigenvalues of $H(h)$ and by $\left\{u_{j}(x ; h)\right\}_{j=1}^{\infty}$ an orthonormal system of the corresponding eigenfunctions. We further denote by $\chi_{d}(x ; h)$, $d>0$, the characteristic function of the set $\left\{x: b(x)<d h^{-1}\right\}$ and by $\chi_{d}(h)$ the multiplication by $\chi_{d}(x ; h)$. For $M \gg 1$ large enough, we define 


$$
\begin{gathered}
N_{M}(\lambda ; h)=\sum_{\lambda_{j}(h)<\lambda}\left\|\chi_{M}(h) u_{j}\right\|^{2} \\
\hat{N}_{M}(\lambda ; h)=\sum_{\lambda_{j}(h)<\lambda}\left\|\left(1-\chi_{M}(h)\right) u_{j}\right\|^{2},
\end{gathered}
$$

where \|\| denotes the $L^{2}$ norm. By definition, it follows that

$$
N_{E}(h)=N_{M}\left(h^{-2} ; h\right)+\hat{N}_{M}\left(h^{-2} ; h\right)
$$

for $E=1$. The proof of Theorem 2 is reduced to proving the following two main lemmas.

Lemma 2.1. Let $\theta(\lambda ; b)$ be defined by (1.4). Then

$$
N_{M}\left(h^{-1} ; h\right)=2(2 \pi)^{-1} \theta\left(h^{-2} ; h^{-1} b\right)(1+o(1)), \quad h \rightarrow 0,
$$

where the order estimate may depend on $M$.

Lemma 2.2. There exists $h_{0}=h_{0}(M)$ such that if $0<h<h_{0}$, then

$$
\hat{N}_{M}\left(h^{-1} ; h\right) \leqq C M^{-1} \theta\left(h^{-2} ; h^{-1} b\right)
$$

for $C$ independent of $M \gg 1$.

It is easy to see that Theorem 2 follows immediately from Lemmas 2.1 and 2.2 .

Now, we define $\phi_{M}(\lambda ; h), \lambda>0$, by

$$
\phi_{M}(\lambda ; h)=h^{-3 / 2} \sum_{j=0}^{\infty} \int_{G_{j M}} b(x)(\lambda h-(2 j+1) b(x))^{1 / 2} d x
$$

where

$$
G_{j M}=\left\{x: b(x)<\min \left(M h^{-1},(2 j+1)^{-1} \lambda h\right)\right\} .
$$

By definition, $\phi_{M}\left(h^{-2} ; h\right)=\theta\left(h^{-2} ; h^{-1} b\right)$. Lemma 2.1 is proved by applying the tauberian arguments to the following trace formula.

LEMMA 2.3. For any $\delta, 0<\delta \ll 1$, small enough, there exists $h_{1}=$ $h_{1}(\delta, M)$ such that if $0<t<\delta^{-1} h^{2}$ for $0<h<h_{1}$, then

$$
\operatorname{Tr}\left[\chi_{M}(h) \exp (-t H(h))\right]=2(2 \pi)^{-2} \int_{0}^{\infty} \exp (-t \lambda) d \phi_{M}(\lambda ; h)(1+O(\delta)),
$$

where the order estimate may depend on $M$.

We shall prove Lemma 2.1 in Section 3, accepting Lemma 2.3 as proved, and Lemma 2.2 in Section 4. The proof of Lemma 2.3 will be given in Sections 5-7. 


\section{§3. Proof of Lemma 2.1}

3.1. First we prepare two lemmas.

Lemma 3.1. Let $m(\lambda)$ be defined by (1.2). Then

$$
\operatorname{Tr}\left[\chi_{M}(h) \exp (-t H(h))\right] \leqq C m\left(M h^{-1}\right) t^{-3 / 2} .
$$

Proof. We denote by $[\exp (-t H(h))](x, y)$ the integral kernel of $\exp (-t H(h))$. By the Feynmna-Kac-Itô formula ([1], Theorem 2.3), we have

$$
|[\exp (-t H(h))](x, y)| \leqq[\exp (t \Delta)](x, y)=O\left(t^{-3 / 2}\right),
$$

which proves the lemma at once.

It follows from Lemma 3.1 that for $\lambda>0$

$$
N_{M}(\lambda ; h) \leqq C_{1} \operatorname{Tr}\left[\chi_{M}(h) \exp \left(-\lambda^{-1} H(h)\right)\right] \leqq C_{2} m\left(M h^{-1}\right) \lambda^{3 / 2} .
$$

LEMMA 3.2. Let

$$
\sigma_{M}(\lambda ; h)=m\left(\min \left(M h^{-1}, \lambda h\right)\right) .
$$

Then there exists $C>1$ independent of $\lambda>0$ and $h$ such that

$$
C^{-1} \sigma_{M}(\lambda ; h) \lambda^{3 / 2} \leqq \phi_{M}(\lambda ; h) \leqq C \sigma_{M}(\lambda ; h) \lambda^{3 / 2} \text {. }
$$

Proof. Let

$$
\sum_{k M}=\left\{x: b(x)<M h^{-1},(2 k+3)^{-1} \lambda h \leqq b(x)<(2 k+1)^{-1} \lambda h\right\}
$$

for $k \geqq 0$ non-negative integer, and define $\phi_{j k M}(\lambda), k \geqq j \geqq 0$, by

$$
\phi_{j k M}(\lambda ; h)=h^{-3 / 2} \int_{\Sigma k M} b(x)(\lambda h-(2 j+1) b(x))^{1 / 2} d x .
$$

By the definition $(2.3)$ of $\phi_{M}(\lambda ; h)$,

$$
\phi_{M}(\lambda ; h)=\sum_{j=0}^{\infty} \sum_{k=j}^{\infty} \phi_{j k M}(\lambda ; h)=\sum_{k=0}^{\infty} \sum_{j=0}^{k} \phi_{j k M}(\lambda ; h) .
$$

On the set $\sum_{k M}, k \geqq j$, we have

$$
\frac{2(k-j)}{(2 k+1)} \lambda h \leqq \lambda h-(2 j+1) b(x) \leqq \frac{2(k+1-j)}{(2 k+3)} \lambda h
$$

and hence

$$
\phi_{j k M}(\lambda ; h) \geqq C(k+1)^{-1} \lambda^{3 / 2} \text { meas }\left[\sum_{k M}\right]
$$

for $j \leqq[k / 2]$, [ ] being the Gauss notation. This yields the lower bound for $\phi_{M}(\lambda ; h)$. The upper bound is obtained in a similar way. 
Since $\sigma_{M}(\lambda ; h) \leqq m\left(M h^{-1}\right)$, it follows from Lemma 3,2 that

$$
\phi_{M}(\lambda ; h) \leqq C m\left(M h^{-1}\right) \lambda^{3 / 2}, \quad \lambda>0 .
$$

3.2. We shall prove Lemma 2.1, accepting Lemma 2.3 as proved. The proof is essentially the tauberian argument due to Karamata [7]. We follow the arguments in [14], paying a little attention to the $h$-dependence.

Proof of Lemma 2.1. (1) We set

$$
\begin{aligned}
& F(t)=\operatorname{Tr}\left[\chi_{M}(h) \exp (-t H(h))\right]=\int_{0}^{\infty} \exp (-t \lambda) d N_{M}(\lambda ; h), \\
& G(t)=2(2 \pi)^{-2} \int_{0}^{\infty} \exp (-t \lambda) d \phi_{M}(\lambda ; h) .
\end{aligned}
$$

For any $\delta, 0<\delta \ll 1$, let $\rho_{\delta}(\tau) \in C^{\infty}[(0, \infty)), 0 \leqq \rho_{\delta} \leqq 1$, be a function such that $\rho_{\delta}=1$ for $0 \leqq \tau \leqq 1$ and $\rho_{\delta}=0$ for $\tau>1+\delta$. The space of all finite linear combinations of functions $t \rightarrow e^{-t \tau}, \tau>0$, is dense in the Schwartz space $\mathscr{S}([0, \infty))$. Hence we can find a function $\kappa_{\delta}(t) \in C_{0}^{\infty}((0, \infty))$ such that

$$
\left|\hat{\kappa}_{\delta}(\tau)-\rho_{\delta}(\tau)\right| \leqq \delta(1+\tau)^{-2}, \quad \tau \geqq 0,
$$

where $\hat{\kappa}_{\delta}(\tau)$ is the Laplace transform of $\kappa_{\delta}(t)$. We may assume that $\kappa_{\delta}(t)$ is supported in $\left\{t: T_{\delta}^{-1}<t<T_{\delta}\right\}$ for some $T_{\delta}>1$. We have the identities

$$
\begin{gathered}
\int_{0}^{\infty} \kappa_{\delta}(t) F\left(h^{2} t\right) d t=\int_{0}^{\infty} \hat{\kappa}_{\delta}\left(h^{2} \lambda\right) d N_{M}(\lambda ; h) \\
\int_{0}^{\infty} \kappa_{\delta}(t) G\left(h^{2} t\right) d t=2(2 \pi)^{-2} \int_{0}^{\infty} \hat{\kappa}_{\delta}\left(h^{2} \lambda\right) d \phi_{M}(\lambda ; h) .
\end{gathered}
$$

(2) We take and fix $\varepsilon=\varepsilon(\delta), 0<\varepsilon<\delta$, so small that $\varepsilon T_{\delta}<1 / 2$ and

$$
\varepsilon \int_{0}^{\infty}\left|\kappa_{\delta}(t)\right| t^{-3 / 2} d t<\delta .
$$

We now use Lemma 2.3 with $\delta=\varepsilon(\delta)$. By Lemma 3.1 and by the above choice of $\varepsilon$, there exists $h_{2}=h_{2}(\delta, M)$ such that

$$
\left|\int_{0}^{\infty} \kappa_{\delta}(t)\left(F\left(h^{2} t\right)-G\left(h^{2} t\right)\right) d t\right| \leqq C \delta h^{-3} m\left(M^{-1} h\right)
$$

for $0<h<h_{2}$.

(3) By (3.2) and (3.5), we have

$$
\int_{0}^{\infty}\left|\hat{\kappa}_{\delta}\left(h^{2} \lambda\right)-\rho_{\delta}\left(h^{2} \lambda\right)\right| d N_{M}(\lambda ; h) \leqq C \delta h^{-3} m\left(M^{-1} h\right)
$$


and similarly by (3.4),

$$
\int_{0}^{\infty}\left|\hat{\kappa}_{\delta}\left(h^{2} \lambda\right)-\rho_{\delta}\left(h^{2} \lambda\right)\right| d \phi_{M}(\lambda ; h) \leqq C \delta h^{-3} m\left(M^{-1} h\right) .
$$

We combine (3.6)-(3.10) to obtain

$$
\left|\int_{0}^{\infty} \rho_{\delta}\left(h^{2} \lambda\right) d N_{M}(\lambda ; h)-2(2 \pi)^{-2} \int_{0}^{\infty} \rho_{\delta}\left(h^{2} \lambda\right) d \phi_{M}(\lambda ; h)\right| \leqq C \delta h^{-3} m\left(M h^{-1}\right) .
$$

Since $\phi_{M}\left(h^{-2} ; h\right)=\theta\left(h^{-2} ; h^{-1} b\right)$ and $\sigma_{M}\left(h^{-2} ; h\right)=m\left(h^{-1}\right)$, it follows from (A.4) and Lemma 3.2 that

$$
C^{-1} \theta\left(h^{-2} ; h^{-1} b\right) \leqq h^{-3} m\left(M h^{-1}\right) \leqq C \theta\left(h^{-2} ; h^{-1} b\right) .
$$

Thus, we have

$$
N_{M}\left(h^{-2} ; h\right) \leqq 2(2 \pi)^{-2} \theta\left((1+\delta) h^{-2} ; h^{-1} b\right)+C \delta \theta\left(h^{-2} ; h^{-1} b\right)
$$

and

$$
N_{M}\left(h^{-2} ; h\right) \geqq 2(2 \pi)^{-2} \theta\left((1-\delta) h^{-2} ; h^{-1} b\right)-C \delta \theta\left(h^{-2} ; h^{-1} b\right) .
$$

(4) The proof is completed by the following

\section{Lemma 3.3.}

(i ) $\lim _{\delta \downarrow 0} \limsup _{h \rightarrow 0} \theta\left((1+\delta) h^{-2} ; h^{-1} b\right) / \theta\left(h^{-2} ; h^{-1} b\right)=1$

(ii) $\lim _{\delta \downarrow 0} \liminf _{h \rightarrow 0} \theta\left((1-\delta) h^{-2} ; h^{-1} b\right) / \theta\left(h^{-2} ; h^{-1} b\right)=1$.

Proof. We prove (i) only, (ii) is proved similarly. We set

$$
\begin{aligned}
& Y_{1 j}=\left\{x:(2 j+1) b(x)<(1-\sqrt{\delta}) h^{-1}\right\} \\
& Y_{2 j}=\left\{x:(1-\sqrt{\delta}) h^{-1} \leqq(2 j+1) b(x)<(1+\delta) h^{-1}\right\}
\end{aligned}
$$

and write

$$
\theta\left((1+\delta) h^{-2} ; h^{1} b\right)=h^{-3 / 2}\left(\theta_{1 \hat{\delta}}(h)+\theta_{2 \delta}(h)\right),
$$

where

$$
\theta_{h \grave{o}}(h)=\sum_{j=0}^{\infty} \int_{Y_{k j}} b(x)\left((1+\delta) h^{-1}-(2 j+1) b(x)\right)^{1 / 2} d x, \quad 1 \leqq k \leqq 2 .
$$

If $x \in Y_{1 j}$, then

$$
(1+\delta) h^{-1}-(2 j+1) b(x) \leqq(1+\sqrt{\delta})\left(h^{-1}-(2 j+1) b(x)\right)
$$

and hence 


$$
h^{-3 / 2} \theta_{1 \delta}(h) \leqq(1+\sqrt{\delta})^{1 / 2} \theta\left(h^{-2} ; h^{-1} b\right)
$$

If $x \in Y_{2 j}$, then

$$
(1+\delta) h^{-1}-(2 j+1) b(x) \leqq 2 \sqrt{\delta} h^{-1}
$$

and hence

$$
h^{-3 / 2} \theta_{2 \delta}(h) \leqq C \delta^{1 / 4} h^{-2} \theta_{0}(h)
$$

where

$$
\theta_{0}(h)=\sum_{j=0}^{\infty} \int_{G_{j}} b(x) d x, \quad G_{j}=\left\{x:(2 j+1) b(x)<2 h^{-1}\right\} .
$$

By the same arguments as in the proof of Lemma 3.2, we can show that

$$
C^{-1} h^{-1} m\left(h^{-1}\right) \leqq \theta_{0}(h) \leqq C h^{-1} m\left(h^{-1}\right),
$$

which, together with (3.11)-(3.13), proves the lemma.

\section{§4. Proof of Lemma 2.2}

The proof of Lemma 2.2 is divided into several steps.

4.1. We begin by introducing new notations. Let $A(x)=\left(a_{1}(x), a_{2}(x)\right.$, $\left.a_{3}(x)\right)$ be the given magnetic potential. We set $\Pi_{j}(h)=i \partial_{j}+(2 h)^{-1} a_{\jmath}, 1 \leqq$ $j \leqq 3$, so that $\Pi_{j}^{*}(h)=\Pi_{j}(h)$ and

$$
H(h)=\sum_{j=1}^{3} \Pi_{j}^{*}(h) \Pi_{j}(h) .
$$

We define $b_{j k}(x), 1 \leqq j, k \leqq 3$, by

$$
b_{j k}(x)=\partial_{j} a_{k}(x)-\partial_{k} a_{j}(x) .
$$

Then, $\sum_{1 \leqq j, k \leqq 3} b_{j k}(x)^{2}=2 b(x)^{2}$ and

$$
\left[\Pi_{j}(h), \Pi_{k}(h)\right]=\Pi_{j}(h) \Pi_{k}(h)-\Pi_{k}(h) \Pi_{j}(h)=i(2 h)^{-1} b_{j k} .
$$

Lemma 4.1. Write $\Pi_{j}$ for $\Pi_{j}(h)$ and denote by $($,$) the L^{2}$ scalar product. Let $a_{j k}(x), 1 \leqq j, k \leqq 3$, be a real $C^{1}$-smooth function. Then

$$
2 \operatorname{Im}\left(a_{j k} \Pi_{j} \phi, \Pi_{k} \phi\right)=-(2 h)^{-1}\left(a_{j k} b_{j k} \phi, \phi\right)+\left(\Pi_{j} \phi,\left(\partial_{k} a_{j k}\right) \phi\right)-\left(\Pi_{k} \phi,\left(\partial_{j} a_{j k}\right) \phi\right)
$$

for $\phi \in C_{0}^{\infty}\left(R_{x}^{3}\right)$.

Proof. We write

$$
2 \operatorname{Im}\left(a_{j k} \Pi_{\jmath} \phi, \Pi_{k} \phi\right)=i\left(\left(\Pi_{j} a_{j k} \Pi_{k}-\Pi_{k} a_{j k} \Pi_{j}\right) \phi, \phi\right) .
$$

A simple commutator calculus proves the lemma. 
The identity above has been used in [6] to prove the compactness property of resolvents.

4.2. We introduce another new notation

$$
X_{d}(h)=\left\{x: b(x)>d h^{-1}\right\}, \quad d>0 .
$$

By assumption (A.3'), we can choose $h_{3}=h_{3}(M)$ so small that

$$
\left|\nabla b_{j k}(x)\right| \leqq M^{-1} b(x)^{2}, \quad x \in X_{M / 2}(h),
$$

for $0<h<h_{3}$. We now take

$$
a_{j k}(x)=h^{-1} M b_{j k}(x) / b(x)^{2}, \quad x \in X_{M / 2}(h),
$$

in the identity of Lemma 4.1. First we note that $\left|a_{j k}(x)\right| \leqq 2$ and $\left|\nabla a_{j k}(x)\right|$ $\leqq C h^{-1}, C$ being independent of $M$, and that

$$
(2 h)^{-1} \sum_{j=1}^{3} \sum_{k=1}^{3} a_{j k}(x) b_{j k}(x)=h^{-2} M .
$$

Thus it follows from Lemma 4.1 that

$$
(H(h) \phi, \phi) \geqq C h^{-2} M(\phi, \phi)
$$

for $\phi$ supported in $X_{M / 2}(h)$.

4.3. Let $\psi_{M}(x ; h) \in C^{1}\left(R_{x}^{3}\right), 0 \leqq \psi_{M} \leqq 1$, be such that $\psi_{M}=1$ on $X_{M}(h)$ and $\psi_{M}=0$ in $R_{x}^{3} \backslash X_{M / 2}(h)$. By (4.1), we may assume that $\left|\nabla \psi_{M}(x ; h)\right| \leqq C h^{-1}$. As is easily seen, (4.2) is still valid for $\psi_{M} u, u \in \mathscr{D}(H(h)$ ) (=domain of $H(h)$ ). Hence, by a simple commutator calculus, we have

$$
\left(H(h) u, \psi_{M}^{2} u\right) \geqq C h^{-2} M\left(\psi_{M} u, \psi_{M} u\right)-C_{1} h^{-2}(u, u) .
$$

Thus we have proved the following

LEMMA 4.2. Let $u_{j}(x ; h)$ be the normalized eigenfunction associated to the eigenvalue $\lambda_{j}(h)<h^{-2}$ of $H(h)$. There exists $h_{4}=h_{4}(M)$ such that if $0<h<h_{4}$, then

$$
\left\|\left(1-\chi_{M}(h)\right) u_{j}\right\|^{2} \leqq C M^{-1}
$$

for $C$ independent of $M \gg 1$.

4.4. By the same arguments as above, we can construct a real function $V(x ; h)$ with the following properties:

( i ) $\quad(H(h) \phi, \phi)>2^{-1}(H(h) \phi, \phi)+(V \phi, \phi), \quad \phi \in C_{0}^{\infty}\left(R_{x}^{3}\right)$;

(ii) $\quad V(x ; h)=O\left(h^{-2}\right)$; 
(iii) $\quad V(x ; h)=2 h^{-2}, \quad x \in X_{d_{0}}(h), \quad$ for some $d_{0}>0$

for $h$ small enough. We define

$$
W(x ; h)=\max \left(0, h^{-2}-V(x ; h)\right) .
$$

By properties (ii) and (iii), $W(x ; h)=O\left(h^{-2}\right)$ and is supported in $\{x: b(x)<$ $\left.d_{0} h^{-1}\right\}$. By property (i), $N_{E}(h)$ with $E=1$ is majorized by the number of negative eigenvalues of the operator $H(h)-2 W$. By the Rosenbljum-CwikelLieb bound ([1], Theorem 2.15), we have

$$
N_{1}(h) \leqq C \int_{R^{3}} W(x ; h)^{3 / 2} d x \leqq C h^{-3} m\left(d_{0} h^{-1}\right),
$$

which, together with Lemma 4.2 and (3.11), completes the proof of Lemma 2.2 .

\section{§. Bounds on the traces}

The aim here is to prove the following

Lemma 5.1. Let $\phi_{M}(\lambda ; h)$ be defined by (2.3). For any $\delta, 0<\delta \ll 1$, there exists $C_{0}=C_{0}(\delta, M)$ such that if $0<t<\delta^{-1} h^{2}$, then

$$
\operatorname{Tr}\left[\chi_{2 M}(h) \exp (-t H(h))\right] \leqq C_{0} \int_{0}^{\infty} \exp (-2 t \lambda) d \phi_{M}(\lambda ; h) .
$$

Proof. By Lemmas 3.1 and 3.2 and by assumption (A.4), we have only to show that there exists $C=C(\delta, M)$ such that

$$
m\left(M h^{-1}\right) t^{-3 / 2} \leqq C \int_{0}^{\infty} \exp (-2 t \lambda) d\left(\sigma_{M}(\lambda ; h) \lambda^{3 / 2}\right)
$$

for $0<t<\delta^{-1} h^{2}$. By the definition (3.3) of $\sigma_{M}(\lambda ; h), \sigma_{M}(\lambda ; h)=m\left(M h^{-1}\right)$ for $\lambda \geqq M h^{-2}$, and hence

$$
\int_{0}^{\infty} \exp (-2 t \lambda) d\left(\sigma_{M}(\lambda ; h) \lambda^{3 / 2}\right) \geqq m\left(M h^{-1}\right) t^{-3 / 2} \int_{M h^{-2} t}^{\infty} \exp (-2 \tau) d \tau^{3 / 2} .
$$

If $0<t<\delta^{-1} h^{2}$, then the existence of such a constant $C$ follows at once.

For later reference, we here state the following

LeMma 5.2. For any $\delta, 0<\delta \ll 1$, there exists $h_{5}=h_{5}(\delta, M)$ such that if $0<t<\delta^{-1} h^{2}$ for $0<h<h_{5}$, then: 
( i ) $\quad \int_{0}^{\infty} \exp (-t \lambda) d \lambda^{3 / 2}=O\left(t^{-3 / 2}\right) \leqq \delta \int_{0}^{\infty} \exp (-2 t \lambda) d \phi_{M}(\lambda ; h)$

(ii ) $\quad \int_{0}^{\infty} \exp (-t \lambda) d\left(\sigma_{M}(\lambda ; h) \lambda^{3 / 2}\right) \leqq C \int_{0}^{\infty} \exp (-2 t \lambda) d \phi_{M}(\lambda ; h)$.

Proof. Since $m\left(h^{-1}\right) \rightarrow \infty$ as $h \rightarrow 0$, (i) follows from (5.1) and Lemma 3.2. (ii) is similarly proved if we note that

$$
\sigma_{M}(2 \lambda ; h) \lambda^{3 / 2} \leqq C \phi_{M}(\lambda ; h), \quad \lambda>\Lambda_{3} h^{-1},
$$

which follows from (A.4) and Lemma 3.2.

\section{§6. Proof of Lemma 2.3}

The proof is divided into several steps.

(1) Let $C_{0}(\delta, M)$ be as in Lemma 5.1. We take and fix $\varepsilon=\varepsilon(\delta, M)$, $0<\varepsilon<\delta$, so small that

$$
\varepsilon^{2} C_{0}(\delta, M)<\delta^{2} .
$$

By assumption (A.3'), we can take $R_{\varepsilon}$ so large that

$$
|b(x)|>\varepsilon^{-2}, \quad|\nabla b(x)|<\varepsilon^{2} b(x)^{2}
$$

for $|x|>R_{\varepsilon}-1$. If $h_{6}=h_{6}(\delta, M)$ is chosen small enough, then the region

$$
\Omega_{M \delta}(h)=\left\{x:|x|>R_{\varepsilon}, b(x)<M h^{-1}\right\}
$$

is not empty for $0<h<h_{6}$.

Lemma 6.1. Let $R_{\varepsilon}$ be as above. There exists $h_{7}=h_{7}(\delta, M)$ such that if $0<t<\delta^{-1} h^{2}$ for $0<h<h_{7}$, then

$$
\int_{|x| \leqq R_{s}}[\exp (-2 t H(h))](x, x) d x \leqq \delta \int_{0}^{\infty} \exp (-2 t \lambda) d \phi_{M}(\lambda ; h) .
$$

The above lemma is an immediate consequence of Lemma 5.2 -(i), because the integral on the left side is of order $O\left(t^{-3 / 2}\right)$ by (3.1). Our next task is to evaluate the integration of $[\exp (-2 t H(h))](x, x)$ over $\Omega_{M \delta}(h)$.

(2) Let $A(x)=\left(a_{1}(x), a_{2}(x), a_{3}(x)\right)$ be the given magnetic potential. We fix $z \in \Omega_{M \hat{\delta}}(h)$. By the Taylor expansion,

$$
a_{j}(x)=a_{j}(z)+\left\langle\left(\nabla a_{j}\right)(z), x-z\right\rangle+r_{j z}(x), \quad 1 \leqq j \leqq 3,
$$

where $\langle$,$\rangle denotes the scalar product in R^{3}$, and

$$
r_{j z}(x)=(2 !)^{-1} \sum_{|\alpha|=2}\left[\int_{0}^{1}\left(\partial_{x}^{\alpha} a_{j}\right)(z+s(x-z)) d s\right](x-z)^{\alpha} .
$$


Set $R_{z}(x)=\left(r_{1 z}(x), r_{2 z}(x), r_{3 z}(x)\right)$. By definition, $B(x)=\nabla \times A(x)$ and hence we can find a real function $g_{z}(x)$ such that

$$
A(x)=B(z) \times x+R_{z}(x)+\nabla g_{z}(x) .
$$

We now define

$$
\begin{aligned}
& H_{0 z}(h)=\left(i \nabla+(2 h)^{-1} B(z) \times x\right)^{2} \\
& H_{z}(h)=\left(i \nabla+(2 h)^{-1}\left(B(z) \times x+R_{z}(x)\right)\right)^{2} .
\end{aligned}
$$

Let $U_{z}(h)$ be the multiplication by $\exp \left(i(2 h)^{-1} g_{z}(x)\right)$, so that $U_{z}(h): L^{2}\left(R_{x}^{3}\right)$ $\rightarrow L^{2}\left(R_{x}^{3}\right)$ is unitary and

$$
H(h)=U_{z}(h) H_{z}(h) U_{z}^{*}(h) .
$$

(3) Let $\psi(x) \in C_{0}^{\infty}\left(R_{x}^{3}\right), 0 \leqq \psi \leqq 1$, be a function such that $\psi(x)$ is supported in $|x|<1$ and $\psi=1$ on $|x| \leqq 1 / 2$. For $z \in \Omega_{M \delta}(h)$, we set

$$
\psi_{\delta z}(x ; h)=\psi\left(\varepsilon h^{-1 / 2} b(z)^{1 / 2}(x-z)\right),
$$

where $\varepsilon=\varepsilon(\delta, M)$ is fixed in step (1). Then the following relation can be easily established:

$$
\begin{aligned}
\psi_{\delta z} \exp \left(-t H_{z}(h)\right)= & \exp \left(-t H_{0 z}(h)\right) \psi_{\delta z} \\
& +\int_{0}^{t} \exp \left(-(t-s) H_{0 z}(h)\right) L_{\delta z}(h) \exp \left(-s H_{z}(h)\right) d s,
\end{aligned}
$$

where

$$
L_{\delta z}(h)=\left[H_{0 z}(h), \psi_{\delta z}\right]+\psi_{\delta z}\left(H_{0 z}(h)-H_{z}(h)\right) .
$$

We now let the above relation operate on the normalized eigenfunction $u_{j}(x ; h)$ associated with the eigenvalue $\lambda_{j}(h)$. Then we obtain

$$
\exp \left(-t \lambda_{j}(h)\right) u_{j}(z ; h)=\sum_{k=0}^{2} v_{k \jmath}(t, z ; h),
$$

where

$$
\begin{aligned}
& v_{0 j \delta}=\left.\left(U_{z}(h) \exp \left(-t H_{0 z}(h)\right) \psi_{\delta z} U_{z}^{*}(h) u_{j}\right)(x ; h)\right|_{x=z} \\
& v_{1 j \delta}=\left.\int_{0}^{t / 2} \exp \left(-s \lambda_{j}(h)\right)\left(T_{\delta z}(t-s ; h) u_{j}\right)(x ; h)\right|_{x=z} d s \\
& v_{2 j \delta}=\left.\int_{t / 2}^{t} \exp \left(-s \lambda_{j}(h)\right)\left(T_{\delta z}(t-s ; h) u_{j}\right)(x ; h)\right|_{x=z} d s \\
& T_{\delta z}(t ; h)=U_{z}(h) \exp \left(-t H_{0 z}(h)\right) L_{\delta z}(h) U_{z}^{*}(h) .
\end{aligned}
$$

(4) The next lemma, together with Lemma 6.1, proves Lemma 2.3. 
Lemma 6.2. Let $\Omega_{M \grave{\delta}}(h)$ be defined by (6.3). Define

$$
I_{k \delta}(t ; h)=\left.\sum_{j=1}^{\infty} \int_{\Omega_{M \delta}(h)} v_{k j \delta}(t, z ; h)\right|^{2} d z, \quad 0 \leqq k \leqq 2 .
$$

There exists $h_{8}=h_{8}(\delta, M)$ such that if $0<t<\delta^{-1} h^{2}$ for $0<h<h_{8}$, then

$$
\begin{aligned}
& I_{0 \hat{\delta}}(t ; h)=2(2 \pi)^{-2} \int_{0}^{\infty} \exp (-2 t \lambda) d \phi_{M}(\lambda ; h)(1+O(\delta)) \\
& I_{k \delta}(t ; h)=\int_{0}^{\infty} \exp (-2 t \lambda) d \phi_{M}(\lambda ; h) O\left(\delta^{2}\right), \quad 1 \leqq k \leqq 2,
\end{aligned}
$$

where the order relations may depend on $M$.

We will prove this lemma in Section 7.

\section{§7. Completion of proof of Lemma 2.3}

In this section we shall complete the proof of Lemma 2.3 by proving Lemma 6.2.

7.1. We write $\hat{B}(z)=B(z) / b(z)$, so that $|\hat{B}(z)|=1$ if $b(z) \neq 0$. The integral kernel of $\exp \left(-t H_{0 z}(h)\right), z \in \Omega_{M \delta}(h)$, can be explicitly calculated ([1], [12]);

$$
\begin{aligned}
& \left.\left[\exp \left(-t H_{0 z} h\right)\right)\right](x, y) \\
& \quad=(4 \pi t)^{-3 / 2} F_{0}\left(h^{-1} b(z) t\right) \exp \left(-F_{1 z}(x-y, t ; h)-i F_{2 z}(x, y ; h)\right),
\end{aligned}
$$

where $F_{0}(s)=s(\sinh s)^{-1}, s>0$,

$$
\begin{aligned}
F_{1 z} & =(4 t)^{-1}\left\{|\langle\hat{B}(z), x-y\rangle|^{2}+\left(h^{-1} b(z) t\right) \operatorname{coth}\left(h^{-1} b(z) t\right)|B(z) \times(x-y)|^{2}\right\} \\
F_{2 z} & =2^{-1}\left\langle h^{-1} B(z) \times x, y\right\rangle .
\end{aligned}
$$

Let

$$
P_{z}(h)=\left(P_{1 z}(h), P_{2 z}(h), P_{3 z}(h)\right)=i \nabla_{y}+(2 h)^{-1} B(z) \times y .
$$

Then $P_{j z}^{*}(h)=P_{j z}(h), 1 \leqq j \leqq 3$, and also $H_{0 z}(h)=\sum_{j=1}^{3} P_{j z}(h)^{2}$, if we regard $H_{0 z}(h)$ as a differential operator acting on functions of $y$-variables.

\section{Lemma 7.1.}

(i ) $\left|\left[\exp \left(-t H_{0 z}(h)\right)\right](z, y)\right| \leqq C t^{-3 / 2} \exp \left(-\frac{2}{3} h^{-1} b(z) t\right) \exp \left(-C^{-1} t^{-1}|y-z|^{2}\right)$.

(ii) $\left|P_{j z}(h) \overline{\left.\exp \left(-t H_{0 z}(h)\right)\right](z, y)}\right|$

$$
\leqq C t^{-2} \exp \left(-\frac{2}{3} h^{-1} b(z) t\right) \exp \left(-C^{-1} t^{-1}|y-z|^{2}\right), \quad 1 \leqq j \leqq 3 .
$$

The above lemma follows immediately from the representation for $\left[\exp \left(-t H_{0 z}(h)\right)\right](x, y)$. 
LEMMA 7.2.

$$
\begin{aligned}
& \int\left|\left[\exp \left(-t H_{0 z}(h)\right)\right](z, y)\right|^{2} d y \\
& \quad=(2 \pi)^{-2} h^{-1} b(z) \sum_{j=0}^{\infty} \int_{-\infty}^{\infty} \exp \left(-2 t\left(\xi^{2}+(2 j+1) h^{-1} b(z)\right)\right) d \xi,
\end{aligned}
$$

where the integration with no domain attached is taken over the whole space $R^{3}$.

Proof. If we write

$$
c_{1}|\langle\hat{B}(z), y\rangle|^{2}+c_{2}|\hat{B}(z) \times y|^{2}=\langle A y, y\rangle, \quad c_{j}>0,
$$

for some $3 \times 3$ positive matrix $A$, then

$$
\int \exp (-\langle A y, y\rangle) d y=(\operatorname{det} A)^{-1 / 2} \pi^{3 / 2}=c_{1}^{-1 / 2} c_{2}^{-1} \pi^{3 / 2} .
$$

We denote by $J_{0}(t, z ; h)$ the integral under consideration and use the above formula with

$$
c_{1}=(2 t)^{-1}, \quad c_{2}=(2 h)^{-1} b(z) \operatorname{coth}\left(h^{-1} b(z) t\right) .
$$

Then we have

$$
J_{0}(t, z ; h)=2^{-7 / 2} \pi^{-3 / 2} t^{-1 / 2} h^{-1} b(z)\left(\sinh \left(2 h^{-1} b(z) t\right)\right)^{-1} .
$$

The proof is completed by making use of the following two identities:

$$
\begin{aligned}
& t^{-1 / 2}=2^{1 / 2} \pi^{-1 / 2} \int_{-\infty}^{\infty} \exp \left(-2 t \xi^{2}\right) d \xi ; \\
& (\sinh s)^{-1}=2 \sum_{j=0}^{\infty} \exp (-(2 j+1) s), \quad s>0
\end{aligned}
$$

For later reference, we here make a brief comment on the integral $J_{0}(t, z ; h)$.

Remark 7.1. If we note that $\lim _{s \downarrow 0} s(\sinh s)^{-1}=1$, the function $J_{0}(t, z ; h)$ is well-defined for all $z \in R^{3}$. In particular, $J_{0}(t, z ; h)$ can be defined for $z$ with $b(z)=0$ and also we can easily see that $J_{0}(t, z ; h)=O\left(t^{-3 / 2}\right), z \in R^{3}$, uniformly in $h$.

7.2. First we prove (6.5).

Proof of (6.5). By the Parseval relation, we have

$$
\sum_{j=1}^{\infty}\left|v_{0 j \delta}(t, z ; h)\right|^{2}=\int \psi_{\delta z}(y ; h)^{2}\left|\left[\exp \left(-t H_{0 z}(h)\right)\right](z, y)\right|^{2} d y .
$$


We write the above integral as $J_{0}(t, z ; h)+J_{\delta}(t, z ; h)$, where $J_{0}$ is defined by (7.2) and

$$
J_{\delta}(t, z ; h)=\int\left(\psi_{\delta z}(y ; h)^{2}-1\right)\left|\left[\exp \left(-t H_{0 z}(h)\right)\right](z, y)\right|^{2} d y .
$$

By Remark 7.1, $J_{0}(t, z ; h)$ is well-defined over the whole space $R_{z}^{3}$. We recall the definition (2.3) of $\phi_{M}(\lambda ; h)$. Then, by Lemma 7.2 , we can write

$$
\int_{b(z)<M h-1} J_{0}(t, z ; h) d z=2(2 \pi)^{-2} \int_{0}^{\infty} \exp (-2 t \lambda) d \phi_{M}(\lambda ; h) .
$$

By Remark 7.1, the integral of $J_{0}(t, z ; h)$ over $|z| \leqq R_{\varepsilon}$ is of order $O\left(t^{-3 / 2}\right)$. Hence, by Lemma 5.2-(i), we can choose $h_{9}=h_{9}(\delta, M)$ so small that if $0<$ $t<\delta^{-1} h^{2}$ for $0<h<h_{9}$, then

$$
\int_{\Omega_{M \delta}(h)} J_{0}(t, z ; h) d z=2(2 \pi)^{-2} \int_{0}^{\infty} \exp (-2 t \lambda) d \phi_{M}(\lambda ; h)(1+O(\delta)) .
$$

We evaluate the integral of $J_{\delta}(t, z ; h)$. By the definition of $\psi_{\delta z}(y ; h)$,

$$
|y-z| \geqq(2 \varepsilon)^{-1} h^{1 / 2} b(z)^{-1 / 2}
$$

on the support of $\psi_{\delta z}(y ; h)^{2}-1$. Hence it follows from Lemma 7.1-(i) that

$$
\begin{gathered}
\left|\left[\exp \left(-t H_{0 z}(h)\right)\right](z, y)\right|^{2}=|y-z|^{-3}|y-z|^{3}\left|\left[\exp \left(-t H_{0 z}(h)\right)\right](z, y)\right|^{2} \\
\leqq C \varepsilon^{3} h^{-3 / 2} b(z)^{3 / 2} t^{-3 / 2} \exp \left(-h^{-1} b(z) t\right) \exp \left(-C^{-1} t^{-1}|y-z|^{2}\right) .
\end{gathered}
$$

Therefore, we have

$$
J_{\delta}(t, z ; h) \leqq C \varepsilon^{3} h^{-3 / 2} b(z)^{3 / 2} \exp \left(-h^{-1} b(z) t\right) .
$$

We here recall the definition (3.3) of $\sigma_{M}(\lambda ; h)$. Then we have

$$
\int_{\Omega_{M \delta}(h)} J_{\delta}(t, z ; h) d z \leqq C \varepsilon^{3} \int_{0}^{\infty} \exp (-t \lambda) d\left(\sigma_{M}(\lambda ; h) \lambda^{3 / 2}\right) .
$$

This, together with Lemma 5.2-(ii), completes the proof of (6.5).

7.3. We regard $L_{\delta z}(h)$ as a differential operator acting on functions of $y$-variables. To prove (6.6), we have to evaluate the function

$$
K_{\delta}(z, y, t ; h)=L_{\delta z}^{*}(h)\left[\overline{\left.\exp \left(-t H_{0 z}(h)\right)\right](z, y)} .\right.
$$

Lemma 7.3. Let $f(x)>0, x \in R_{x}^{3}$, be a function with the property $|\nabla f(x)|$ $\leqq \beta f(x)^{1+\rho}, \rho>0$. If $\gamma=\rho \beta|y-x| f(x)^{\rho}<1$, then

$$
(1+\gamma)^{-1 / \rho} f(x) \leqq f(y) \leqq(1-\gamma)^{-1 / \rho} f(x)
$$


Proof. Set $g(s)=f(x+s(y-x) /|y-x|)$, so that $g(0)=f(x)$ and $g(|y-x|)=f(y)$. By assumption,

$$
-\beta g(s)^{1+\rho} \leqq g^{\prime}(s) \leqq \beta g(s)^{1+\rho} .
$$

The lemma is verified by solving these differential inequalities.

Now, assume that $|y-z|<\varepsilon^{-1} h^{1 / 2} b(z)^{-1 / 2}$ for $z \in \Omega_{M \delta}(h)$. Then, by the choice of $R_{\varepsilon}$, it follows from (6.2) that $|y|>R_{\varepsilon}-1$ and $|y-z| \leqq \varepsilon^{-1} M^{1 / 2} b(z)^{-1}$, because $b(z)^{1 / 2} \leqq M^{1 / 2} h^{-1 / 2}$ for $z \in \Omega_{M \delta}(h)$. Furthermore, $|\nabla b(y)|<\varepsilon^{2} b(y)^{2}$ by (6.2) again. Thus, we have by Lemma 7.3 with $\rho=1$ that

$$
2^{-1} b(z) \leqq b(y) \leqq 2 b(z),
$$

if $\varepsilon$ is chosen so small that $\varepsilon M^{1 / 2}<1 / 2$.

Let $r_{\jmath z}(y), 1 \leqq j \leqq 3$, be defined by (6.4). If $|y-z| \leqq \varepsilon^{-1} h^{1 / 2} b(z)^{-1 / 2}$ for $z \in \Omega_{M \hat{o}}(h)$, then it follows from (7.4) and (A.3') that

$$
\begin{aligned}
& h^{-1}\left|r_{j z}(y)\right| \leqq C \varepsilon h^{-1} b(z)|y-z| \\
& h^{-1}\left|\nabla r_{j z}(y)\right| \leqq C \varepsilon^{2} h^{-3 / 2} b(z)^{3 / 2}|y-z|
\end{aligned}
$$

for $C$ dependning on $M$. Let $P_{j z}(h), 1 \leqq j \leqq 3$, be defined by (7.1). The operator $L_{o z}^{*}(h)$ takes the form

$$
L_{\delta z}^{*}(h)=\sum_{j=1}^{3}\left[c_{j \delta z}(y ; h) P_{j z}(h)+d_{j o z}(y ; h)\right] .
$$

The coefficients $c_{j \tilde{z} z}$ and $d_{j \delta \tilde{z}}$ are supported in $\left\{y:|y-z|<\varepsilon^{-1} h^{1 / 2} b(z)^{-1 / 2}\right\}$ and satisfy the estimates;

$$
\begin{aligned}
& \left|c_{j \delta z}(y ; h)\right| \leqq C \varepsilon h^{-1} b(z)|y-z| \\
& \left|d_{j \delta z}(y ; h)\right| \leqq C \varepsilon h^{-3 / 2} b(z)^{3 / 2}|y-z|,
\end{aligned}
$$

which follow from (7.5), (7.6) and

$$
\left|\partial_{y}^{\alpha} \psi_{\delta z}\right| \leqq C_{\alpha}\left[\varepsilon h^{-1 / 2} b(z)^{1 / 2}\right]^{|\alpha|+1}|y-z|, \quad|\alpha| \geqq 1 .
$$

Thus we combine (7.7) and (7.8) with Lemma 7.1 to obtain the following

Lemma 7.4. Let $K_{\dot{\delta}}(z, y, t ; h), z \in \Omega_{M \delta}(h)$, be defined by (7.3). Then, $K_{\dot{o}}$ is supported in $\left\{y:|y-z|<\varepsilon^{-1} h^{1 / 2} b(z)^{-1 / 2}\right\}$ as a function of y-variables and

$$
\left|K_{\delta}\right| \leqq C \varepsilon h^{-1} b(z) t^{-2} \exp \left(-\frac{1}{2} h^{-1} b(z) t\right)\left[|y-z| \exp \left(-C^{-1} t^{-1}|y-z|^{2}\right)\right] .
$$

7.4. We shall prove (6.6).

Proof of (6.6). (i) First we deal with the case $k=1$. By the Schwarz 
inequality and by the Parseval relation, it follows from Lemma 7.4 that

$$
\begin{aligned}
\sum_{j=1}^{\infty}\left|v_{1 j \delta}(t, z ; h)\right|^{2} & \leqq t \int_{0}^{t / 2} \int \mid\left[\left.K_{\delta}(z, y, t-s ; h)\right|^{2} d y d s\right. \\
& \leqq C \varepsilon^{2} h^{-2} b(z)^{2} t^{1 / 2} \exp \left(-\frac{1}{2} h^{-1} b(z) t\right) .
\end{aligned}
$$

Furthermore, by Lemma 5.2-(ii), we have

$$
\begin{aligned}
I_{1 \delta}(t ; h) & \leqq C \varepsilon^{2} \int_{0}^{\infty} \lambda^{1 / 2} t^{1 / 2} \exp \left(-\frac{1}{2} t \lambda\right) d\left(\sigma_{M}(\lambda ; h) \lambda^{3 / 2}\right) \\
& =\int_{0}^{\infty} \exp (-2 t \lambda) d \phi_{M}(\lambda ; h) O\left(\delta^{2}\right) .
\end{aligned}
$$

This proves (6.6) with $k=1$.

(ii) Next we deal with the case $k=2$. We start with the estimate

$$
\left|v_{2 j \delta}(t, z ; h)\right| \leqq \exp \left(-\frac{1}{2} t \lambda_{j}(h)\right) \int_{0}^{t / 2} \int\left|K_{\delta}(z, y, s ; h)\right|\left|u_{j}(y)\right| d y d s .
$$

We make a change of variables: $(y, s) \rightarrow(v, \tau)$, where

$$
y=z+h^{1 / 2} b(z)^{-1 / 2} \tau^{1 / 2} v, \quad s=h b(z)^{-1} \tau .
$$

The Jacobian $\partial(y, s) / \partial(v, \tau)$ can be easily calculated:

$$
|\partial(y, s) / \partial(v, \tau)|=h^{5 / 2} b(z)^{-5 / 2} \tau^{3 / 2}
$$

and also by Lemma 7.4, we have

$$
\left|K_{\delta}\right| \leqq C \varepsilon h^{-5 / 2} b(z)^{5 / 2} \tau^{-3 / 2} F_{\delta}(v, \tau)
$$

in the $(v, \tau)$-coordinates, where

$$
F_{\delta}(v, \tau)=\exp \left(-\frac{1}{2} \tau\right)|v| \exp \left(-C^{-1}|v|^{2}\right) \chi_{0}\left(\varepsilon \tau^{1 / 2} v\right)
$$

and $\chi_{0}$ is the characteristic function of the set $\{x:|x| \leqq 1\}$. Thus we have

$$
\left|v_{2 \jmath \delta}(t, z ; h)\right| \leqq C \exp \left(-\frac{1}{2} t \lambda_{j}(h)\right) \int_{0}^{\infty} \int F_{\delta}(v, \tau)\left|w_{j}(z, v, \tau ; h)\right| d v d \tau
$$

where

$$
w_{j}(z, v, \tau ; h)=u_{j}\left(z+h^{1 / 2} b(z)^{-1 / 2} \tau^{1 / 2} v ; h\right) .
$$

Set $\zeta=z+h^{1 / 2} b(z)^{-1 / 2} \tau^{1 / 2} v$. If $z \in \Omega_{M \delta}(h)$ and $\left|\tau^{1 / 2} v\right| \leqq \varepsilon^{-1}$, then it follows from (6.2) that $|\zeta|>R_{\varepsilon}-1$ and $|\zeta-z| \leqq \varepsilon^{-1} M^{1 / 2} b(z)^{-1}$. Furthermore, by (6.2) again, $|\nabla b(\zeta)|<\varepsilon^{2} b(\zeta)^{2}$. Hence by Lemma 7.3 with $\rho=1$, we have $b(\zeta)$ $\leqq 2 b(z) \leqq 2 M h^{-1}$. The Jacobian $\partial(\zeta) / \partial(z)$ satisfies 


$$
|\partial(\zeta) / \partial(z)|=1+O(\varepsilon)=1+O(\delta) .
$$

Thus we have

$$
\int_{b(z)<M h-1}\left|w_{j}(z, v, \tau ; h)\right|^{2} d z \leqq C \int_{b(\zeta)<2 M h-1}\left|u_{j}(\zeta ; h)\right|^{2} d \zeta
$$

for $C$ independent of $(v, \tau),\left|\tau^{1 / 2} v\right|<\varepsilon^{-1}$, and hence

$$
I_{2 \delta}(t ; h) \leqq C \varepsilon^{2} \operatorname{Tr}\left[\chi_{2 M}(h) \exp (-t H(h))\right]
$$

By the choice of $\varepsilon((6.1))$ and by Lemma 5.1, we have

$$
I_{2 \delta}(t ; h)=\int_{0}^{\infty} \exp (-2 t \lambda) d \phi_{M}(\lambda ; h) O\left(\delta^{2}\right) .
$$

This proves (6.6) with $k=2$.

\section{$\S 8$. Sketch of proof of Theorem 1}

In the present section we assume (A.1)-(A.4), and give a sketch for the proof of Theorem 1 . This theorem can be more easily proved than Theorem 2, because we do not need to consider the localized trace as in the proof of Theorem 2.

8.1. We first establish the bound on the trace $\operatorname{Tr}[\exp (-t H)]$. We start with Lemma 4.1 with $h=1$. We fix $R_{0}$ so that $b(x) \geqq 1$ for $|x| \geq R_{0}$ and take

$$
a_{j k}(x)=-b_{j k}(x) / b(x), \quad 1 \leqq j, k \leqq 3,
$$

for $|x|>R_{0}$. As is easily seen, $\left|a_{j k}(x)\right|=O(1)$ and $\left|\nabla a_{j k}(x)\right|=o\left(b(x)^{1 / 2}\right),|x|$ $\rightarrow \infty$, by (A.3). Hence it follows from Lemma 4.1 that $(H \phi, \phi) \geqq C(b \phi, \phi)$ for $\phi \in C_{0}^{\infty}\left(|x|>R_{0}\right)$ and therefore we have

$$
(H \phi, \phi) \geqq 2^{-1}(H \phi, \phi)+C((b-1) \phi, \phi), \quad \phi \in C_{0}^{\infty}\left(R_{x}^{3}\right),
$$

with another constant $C>0$. With this constant $C$, we define the operator $H_{0}$ by $H_{0}=-2^{-1} \Delta+C b$. By (A.4), $m(\lambda)$ is of algebraic growth as $\lambda \rightarrow \infty$; $m(\lambda)=O\left(\lambda^{K}\right)$ for some $K>0$, and by the Rosenbljum-Cwikel-Lieb bound ([9], Theorem XIII.12), the number of eigenvalues less than $\lambda$ of $H_{0}$ is majorized by $C m(\lambda) \lambda^{3 / 2}$. This implies that $\exp \left(-t H_{0}\right), t>0$, is of trace class and that

$$
\operatorname{Tr}\left[\exp \left(-t H_{0}\right)\right] \leqq C \int_{0}^{\infty} \exp (-t \lambda) d\left(m(\lambda) \lambda^{3 / 2}\right)
$$


Hence, by the Feynman-Kac-Itô formula, it follows from (8.1) that $\exp (-t H)$, $t>0$, is of trace class and

$$
\operatorname{Tr}[\exp (-t H)] \leqq C \operatorname{Tr}\left[\exp \left(-t H_{0}\right)\right], \quad 0<t<1 .
$$

By the same arguments as in the proof of Lemma 3.2, we can prove that

$$
C^{-1} m(\lambda) \lambda^{3 / 2} \leqq \theta(\lambda) \leqq C m(\lambda) \lambda^{3 / 2}, \quad \lambda>0 .
$$

Thus we obtain the following bound on the trace:

$$
\operatorname{Tr}[\exp (-t H)] \leqq C \int_{0}^{\infty} \exp (-t \lambda) d \theta(\lambda), \quad 0<t<1 .
$$

8.2. Let $\psi(x) \in C_{0}^{\infty}\left(R_{x}^{3}\right)$ be as in step (3) of Section 6. Making use of the bound above and of the cut-off function

$$
\psi_{i z}(x)=\psi\left(\delta b(z)^{1 / 2}(x-z)\right),
$$

we can obtain the following asymptotic formula:

$$
\operatorname{Tr}[\exp (-t H)]=2(2 \pi)^{-2} \int_{0}^{\infty} \exp (-t \lambda) d \theta(\lambda)(1+o(1)), \quad t \rightarrow 0 .
$$

The same arguments as in the proof of Lemma 3.3 prove that $\theta(\lambda)$ satisfies the conditions of Karamata's tauberian theorem. Thus the proof of Theorem 1 is completed by applying this tauberian theorem to the asymptotic trace formula.

Added in proof. During the submission to the journal, the author knew that Colin de Verdiere obtained a similar result, including the $n$ dimensional case, by a different method based on the min-max principle.

Y. Colin de Verdiere; L'asymptotique de Weyl pour les bouteilles magnétiques, Commun. Math. Phys., 105 (1986), 327-335.

\section{REFERENCES}

[1] J. Avron, I. Herbst and B. Simon, Schrödinger operators with magnetic fields, I, general interactions, Duke Math. J., 45 (1978), 847-883.

[2] M. Sh. Birman and M. Z. Solomyak, Asymptotic behavior of the spectrum of differential equations, J. Soviet Math., 12 (1979), 247-283.

[ 3 ] J. M. Combes, R. Schrader and R. Seiler, Classical bounds and limits for energy distributions of hamiltonian operators in electromagnetic fields, Ann. Physics, 111 (1978), 1-18.

[4] A. Dufresnoy, Un exemple de champ magnetiques dan $R^{\nu}$, Duke Math. J., 50 (1983), 729-734. 
[5] L. Hörmander, Hyoelliptic second order differential equations, Acta. Math., 119 (1967), 147-171.

[6] A. Iwatsuka, Magnetic Schrödinger operators with compact resolvent, To appear in J. Math. Kyoto Univ.

[7] J. Karamata, Neuer Beweis und Verallgemeinerung der Tauberschen Sätze, J. Reine angew. Math., 164 (1931), 27-39.

[8] G. Metivier, Fonction spectrale et valeurs propres d'une class d'operateurs non elliptiques, Comm. Partial Differ. Eqs., 1 (1976), 467-519.

[ 9 ] M. Reed and B. Simon, Methods of Modern Mathematical Physics, Vol. IV Analysis of Operators, Academic Press, 1978.

[10] G. V. Rosenbljum, Asymptotics of the eigenvalues of the Schrödinger operator, Math. USSR Sb., 22 (1974), 349-371.

[11] M. Schechter, Spectra of Partial Differential Operators, North-Holland, 1971.

[12] B. Simon, Functional Integration and Quantum Physics, Academic Press, 1977.

[13] - - Non-classical eigenvalue asymptotics, J. Funct. Anal., 53 (1983), 84-98.

[14] J. Sjöstrand, On the eigenvalues of a class of hypoelliptic operators, IV, Ann. Inst. Fourier Grenoble, 30 (1980), 109-169.

Department of Applied Physics

Nagoya University Chikusa-ku, Nagoya 464

Japan 